\title{
A Wild Yeast Laboratory Activity: From Isolation to Brewing
}

\author{
Amanda N. Scholes, a,b Erik D. Pollock,b and ㅇeffrey A. Lewis ${ }^{c}$ \\ ${ }^{a}$ Cell and Molecular Biology Program, University of Arkansas, Fayetteville, Arkansas, USA \\ ${ }^{b}$ Stable Isotope Laboratory, University of Arkansas, Fayetteville, Arkansas, USA \\ ${ }^{c}$ Department of Biological Sciences, University of Arkansas, Fayetteville, Arkansas, USA
}

Microbial fermentation is a common form of metabolism that has been exploited by humans to great benefit. Industrial fermentation currently produces a myriad of products ranging from biofuels to pharmaceuticals. About one-third of the world's food is fermented, and the brewing of fermented beverages in particular has an ancient and storied history. Because fermentation is so intertwined with our daily lives, the topic is easily relatable to students interested in real-world applications for microbiology. Here, we describe the curriculum for a guided inquiry-based laboratory course that combines yeast molecular ecology and brewing. The rationale for the course is to compare commercial Saccharomyces cerevisiae yeast strains, which have been domesticated through thousands of generations of selection, with wild yeast, where there is growing interest in their potentially unique brewing characteristics. Because wild yeasts are so easy to isolate, identify, and characterize, this is a great opportunity to present key concepts in molecular ecology and genetics in a way that is relevant and accessible to students. We organized the course around three main modules: isolation and identification of wild yeast, phenotypic characterization of wild and commercial ale yeast strains, and scientific design of a brewing recipe and head-to-head comparison of the performance of a commercial and wild yeast strain in the brewing process. Pre- and postassessment showed that students made significant gains in the learning objectives for the course, and students enjoyed connecting microbiology to a real-world application.

KEYWORDS guided inquiry, laboratory exercise, microbial fermentation, wild yeast, brewing science

\section{INTRODUCTION}

Microbial fermentation is a ubiquitous form of metabolism that has been exploited by humans for thousands of years (I-4). About one-third of the world's food is fermented (5), which of course has massive effects on global and local economies. Fermentation has a particularly rich history in the baking and brewing of alcoholic beverages, with the yeast Saccharomyces cerevisiae being among the oldest domesticated organisms $(3,6)$. While the first beer may have been brewed as long as 13,000 years ago (7), what we would now recognize as modern beer took shape in the Middle Ages, where malted barley was used as a source of fermentable sugars, and hops were used as a bittering agent (8). During this time span, continuous selection of yeast in

Citation Scholes AN, Pollock ED, Lewis JA. 202I. A wild yeast laboratory activity: from isolation to brewing. J Microbiol Biol Educ 22:e00186-21. https://doi.org/I0.1 I28/jmbe.00186-21.

Address correspondence to Department of Biological Sciences, University of Arkansas, Fayetteville, Arkansas, USA. E-mail: lewisja@uark.edu.

Received: 25 June 202I, Accepted: 2 July 202I,

Published: 31 August 202 I the brewing environment selected for a number of traits, including better utilization of wort carbon sources and increased fermentation efficiency. Modern brewing styles emerged from regional differences in brewing, and early brewers selected for yeast strains that complemented their brewing ingredients. For example, while the primary products of yeast fermentation are ethanol and carbon dioxide, a number of secondary products, including esters and fusel alcohols are also produced that have unique flavor and aroma profiles (9). Certain beer styles (e.g., Belgian lambic and German-style hefeweizen) favor high levels of secondary fermentation products, while other styles favor little to none and consider these compounds to be "off flavors" (e.g., many stouts and amber ales). The choice of yeast strain became a critical parameter for brewing design.

While brewers have most frequently used domesticated yeast strains, it is becoming increasingly clear that wild yeast strains are important reservoirs for traits important to industrial fermentations, including brewing (I0). This can include novel metabolic capabilities, such as the ability to ferment complex carbohydrates in wort or the ability to produce novel flavor compounds (II). Because wild yeast strains are so easy to isolate, phenotype, and genotype, this provides a unique opportunity for undergraduates in laboratory courses to engage in open or guided inquiry-based research (I2). As such, we 
designed a guided inquiry laboratory course around the microbiology of brewing and fermentation to provide a real-life application. We organized the course around three main modules: isolation and identification of wild yeast, phenotypic characterization of commercial and wild ale yeast strains, and scientific design of a brewing recipe and head-to-head comparison of the performance of a commercial and wild yeast strain in the brewing process.

\section{Intended audience and prerequisite student knowledge}

This course was designed to provide our senior Biology majors with an upper-level Microbiology laboratory course. This course also provides an opportunity for students to write a research paper that can satisfy our university's writing requirement for graduation. Students should have some knowledge of molecular biology and biochemistry, particularly central metabolism and regulation of gene expression. As an upper-level course, students were required to have taken our sophomore-level Cell Biology and General Genetics courses and one of the associated introductory lab courses as prerequisites. Those laboratory courses introduce students to basic biosafety level (BSL)-I microbiology (aseptic work with S. cerevisiae and/or Escherichia coli), which we highly recommend as a requirement for instructors intending to adopt this course. While not required, we also suggested that our junior-level Prokaryote Biology course (an upper-level Microbiology lecture course) would be helpful.

\section{Learning time}

The laboratory was structured as a three credit-hour full-semester course ( 16 weeks). The class was scheduled to meet twice a week for $3 \mathrm{~h}$, and the approximate length of each lab can be found in the instructor's manual (Appendix 2 in the supplemental material). The majority of learning time and experiments took place in the laboratory. Approximately 2 to $3 \mathrm{~h}$ per week was spent outside class on activities such as collecting wild yeast samples, reading relevant scientific literature, and completing homework and laboratory notebook assignments. We estimate that students spent approximately 4 to $6 \mathrm{~h}$ per week when working on their oral presentations and final research papers.

\section{Learning objectives}

The overall goal of the course is to provide both conceptual learning and hands-on laboratory skills. Following completion of the course, students should be able to:

I. Summarize and discuss primary research literature.

2. Predict where wild yeast can be isolated based on the natural ecology of yeast and explain how one can enrich for yeast from environmental samples.
3. Explain why and how internal transcribed spacer (ITS) sequencing is used to determine fungal species and analyze ITS sequencing data to assign the species of an unknown isolate.

4. Describe the primary and secondary products of yeast fermentation and how differences in fermentative metabolism across yeast strains impact brewing.

5. Analyze yeast phenotypic data for traits relevant to brewing and then use those data to predict brewing outcomes.

6. Explain the role of each ingredient and step in the brewing process and scientifically design and implement a brewing protocol.

\section{PROCEDURE}

While we provide detailed student and instructor instructions in the Appendices, here we will briefly describe the main modules of the course (Fig. I).

\section{Wild yeast isolation and identification}

Yeasts are ubiquitous in the environment and can be found on a number of substrates, ranging from rotting fruit to soil to tree bark (13). For the first part of this course, to isolate wild yeast, students are given materials to sample from nature. Students then place the samples in liquid medium that enriches for budding yeast, and samples showing evidence of fermentation (gas bubbles) are plated to identify colonies consistent with those of yeast, which can be confirmed for the presence of budding yeast via microscopy. Following successful yeast isolation, students are provided with methods to perform DNA extractions, PCR, sequencing of the ITS/5.8S ribosomal DNA locus that is frequently used to differentiate yeast species (14), and BLAST analyses to determine the species of their isolated yeast.

\section{Wild and commercial yeast phenotypic characte- rization}

Students are then paired, and half of the class is charged with phenotypically characterizing different wild S. cerevisiae strains, and the other half of the class will characterize different commercial brewing strains. Generally, student pairs who isolated wild $S$. cerevisiae will work with their own stains, while those who did not isolate $S$. cerevisiae are given the commercial strains. First, the entire group learns how to "mash" malted grains together (which they will need to understand for the following module). The resulting wort from each group is then pooled and autoclaved to generate a standardized "beer media" to characterize all of the strains. Students are provided with protocols for phenotyping characterization, including measuring fermentation rates, performing quantitative PCR ( $q P C R$ ) of mRNA levels for genes known to be responsible for ester and fusel 


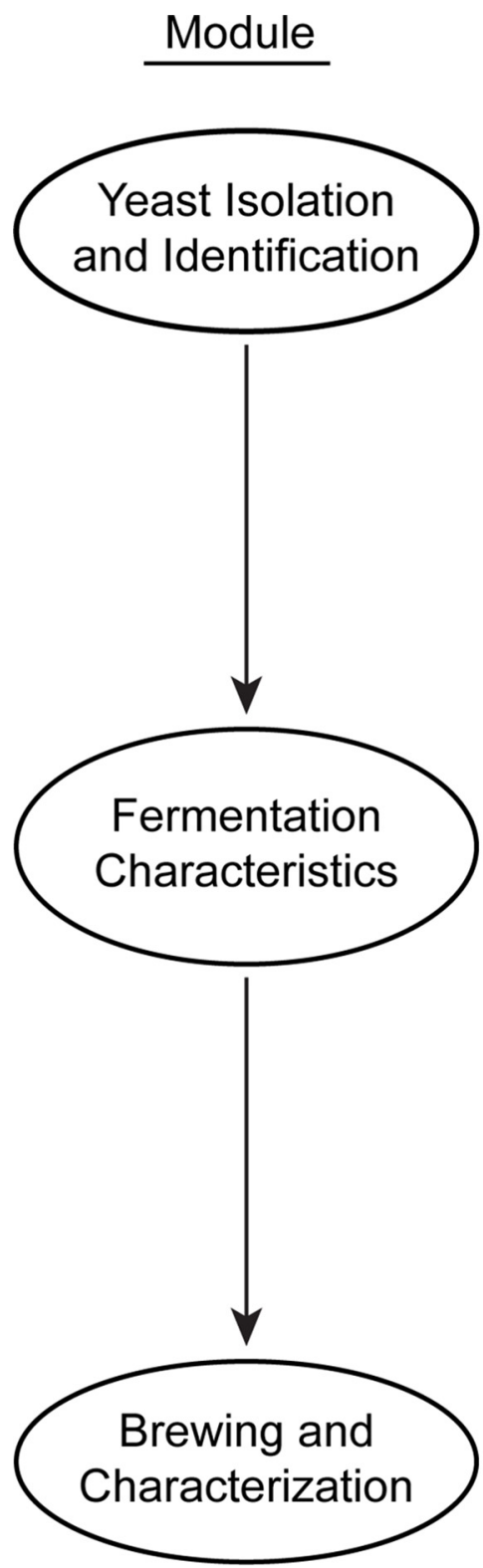

Example Data

ITS PCR

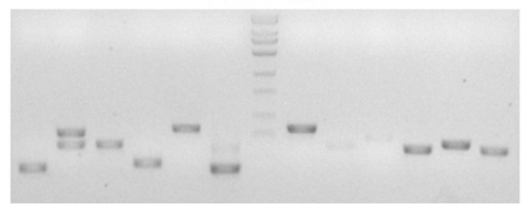

BLAST

\begin{tabular}{|c|c|c|c|c|c|}
\hline Description & Max & Total & Query & & Per. \\
\hline 11 & 1134 & 1134 & $98 \%$ & 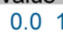 & $100.00 \%$ \\
\hline romyces cerevisiae strain UCDFST:11-194 ITS1 & 1134 & 1134 & $98 \%$ & 0.0 & $100.00 \%$ \\
\hline charomyces cerevisiae strain UCDFST:61-351 ITS1 & 13 & 1134 & $98 \%$ & 0.01 & $100.00 \%$ \\
\hline
\end{tabular}

Fermentation Efficiency

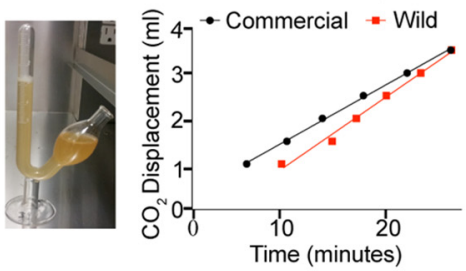

Flavor Compound

Gene Expression
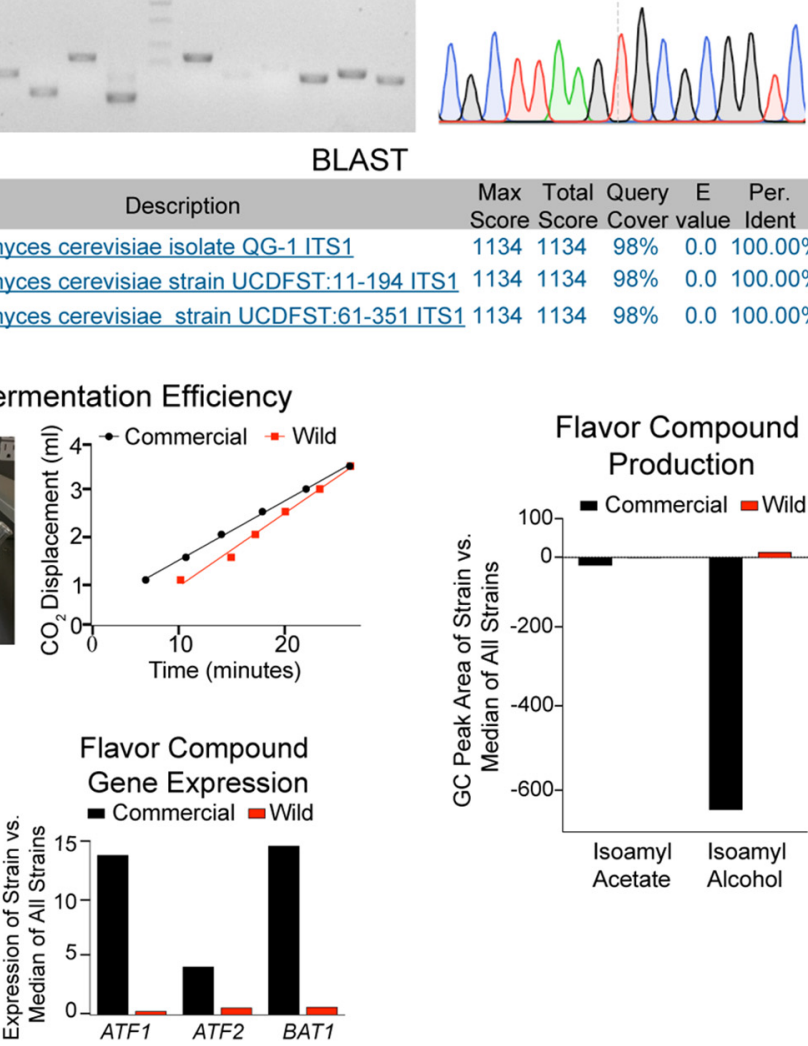

ITS Sequencing

CGCTTAAGTGCGCGGTC 
grains. This leads to conversion of the grain starches into sugars that can be fermented by yeast (with the added wrinkle that alpha and beta amylases are most active at different temperatures, leading to different sugar profiles in the final wort depending on mash temperature). "Roasting" of the malted grains at different temperatures and times leads to lighter or darker malts (with darker malts having fewer active amylases and more Maillard products that are not fermentable). Following mashing, hops are generally added to the resulting sweet wort, which is then boiled. Boiling partially sterilizes the wort and isomerizes hop alpha-acids, leading to characteristic bitterness (with different varieties of hops containing differing amounts of alpha acids and other flavor compounds). Hop iso-alpha-acids also are bacteriostatic against many Gram-positive bacteria $(15,16)$. Finally, the wort is chilled, the yeast is "pitched," and fermentation converts the wort sugars to mainly ethanol and $\mathrm{CO}_{2}$ along with secondary esters and alcohols.

For this course, we used the "brew in a bag" method, where the grains are placed in a bag that is submerged during the mashing process. Following mashing, the bag is simply removed and squeezed to drain the residual sweet wort. Then the sweet wort is brought to a boil for sterilization and hop additions, cooled to allow for yeast pitching, fermented for 3 weeks (typical for many ales), and finally bottle conditioned for 2 weeks. Following brewing, students measured their beers' final gravities (to determine percent attenuation and alcohol percentage), color, bitterness, and secondary flavor compounds. Students also had the option of participating in a voluntary taste test of the final beers. Below is an example of a studentdesigned recipe built around a low ester-producing and highly fermentative yeast strain, and additional recipes can be found in Appendix 7 in the supplemental material.

Recipe: Blood Orange Ginger American Ale

Ingredients

Grain (target original gravity 1.068)

$4.96 \mathrm{lb}$ 2-row U.S. pale malt

$1.3 \mathrm{lb}$ Briess aromatic Munich malt

$0.43 \mathrm{lb}$ flaked wheat

Hops (target international bitterness units [IBU], 86)

$0.58 \mathrm{oz}$ Citra, boiled for $60 \mathrm{~min}$

Additives

I Whirfloc tablet (Irish moss; clarifying agent), boiled for last $5 \mathrm{~min}$

$0.5 \mathrm{oz}$ blood orange extract, boiled for last $5 \mathrm{~min}$

$0.4 \mathrm{oz}$ sliced ginger root, boiled for last $10 \mathrm{~min}$

\section{Mashing}

I. Heat 3.5 gallons of ultra-pure water in stockpot to $67^{\circ} \mathrm{C}$.

2. Add all grain to the "brew bag" within the stockpot and mash at $67^{\circ} \mathrm{C}$ for $60 \mathrm{~min}$.
3. Pull out brew bag and squeeze to drain excess wort. Discard spent grain.

\section{Boiling}

4. Raise mash to a rolling boil.

5. Add Citra hops to a hop bag and add to boiling wort.

6. Incubate for $60 \mathrm{~min}$.

7. With $10 \mathrm{~min}$ left in the boil, add $0.4 \mathrm{oz}$ of sliced ginger root.

8. With $5 \mathrm{~min}$ left, add $0.5 \mathrm{oz}$ of blood orange extract and I Whirfloc tablet.

\section{Fermentation}

9. Cool wort to near room temperature using a wort chiller.

10. Add cooled wort and 65.5 billion yeast cells to a I-gallon fermentation growler.

II. Add airlock and fill with water-diluted Star San sanitizer.

12. Transfer $250 \mathrm{ml}$ of the remaining wort to a graduated cylinder and measure initial gravity with a hydrometer.

13. Place fermentation growlers in a dark area at room temperature for 3 weeks.

Bottle conditioning

14. Add $6.6 \mathrm{ml}$ of $50 \%$ glucose (priming sugar for carbonation) to a sterilized 16-oz amber swing-neck bottle.

15. Auto-siphon the beer into a sterile 16-oz amber bottle.

16. Transfer $250 \mathrm{ml}$ of the remaining beer to a graduated cylinder and measure final gravity with a hydrometer.

17. Incubate at room temperature for 2 weeks in the dark to carbonate the beer.

\section{MATERIALS}

Materials are listed for a class of 24 students working individually for the initial yeast isolation and then in pairs for the subsequent experiments. A full list of the materials, supplies, and equipment are listed in Appendix I in the supplemental material. Materials and recipes for individual modules can be found in Appendix 3.

\section{Student instructions}

The student manual can be found in Appendix 4. Students were required to maintain a lab notebook with detailed rationale, methods, results, and discussion sections. An example lab notebook entry can be given to students to serve as a guide (Appendix 5). The notebook 
was collected three times during the 16-week course. Students were also responsible for preparing a 10-min fermentation-related oral presentation along with a final term paper describing their scientifically designed brewing recipe in journal article format.

\section{Faculty instructions}

Detailed faculty instructions for lab activities can be found in the instructor manual (Appendix 3). Lab lectures and active-learning activities (clicker questions and group discussions) can be found in Appendix 2. Instructor materials for all graded assignments, including associated rubrics, can be found in Appendix 5.

\section{Outcomes and issues for discussion with students}

Because this is a research-based course, anticipated outcomes are not guaranteed. Not all students are guaranteed to isolate yeast for molecular characterization. Those students should be provided with a wild yeast isolate, either from another classmate who isolated more than one unique strain or from the instructor. Likewise, there is no guarantee that the class will isolate enough wild S. cerevisiae strains for subsequent experiments, so the instructors should be prepared to supply wild $S$. cerevisiae strains as a backup. Wild yeast strains can be ordered from the Agricultural Research Service (ARS) Culture Collection (https://nrrl. ncaur.usda.gov), but the corresponding author (Jeff Lewis) is happy to send wild $S$. cerevisiae strains upon request. It is helpful to cryopreserve all positively screened wild S. cerevisiae strains so that they can be used in future classes if necessary. Yeast can be cryopreserved by adding sterile glycerol to a final concentration of $20 \%$ ( $\mathrm{vol} / \mathrm{vol}$ ) to an overnight culture and storing at $-80^{\circ} \mathrm{C}$.

\section{Suggestions for determining student learning}

Pre- and postlaboratory exams and surveys (Appendix 6) were administered to students. The 15-question exam consisted of an equal number of multiple choice, true-false, and short answer questions. The 5-question survey measured student perceptions of proficiency using a Likert-like scale. We did not use quizzes, midterms, or a final exam to assess student learning, although those could certainly be implemented. The ability to summarize and discuss the primary literature was assessed via homework assignments and a short (10- to 12 min) oral presentation. For each module, laboratory notebooks were graded to assess student learning. A final paper in the form of a primary research article was used as an additional summative assessment of student learning.

\section{Safety issues}

All work with unknown organisms must be performed under BSL-2 conditions. Students must demonstrate com- petency with BSL-I safety procedures before working with unknown samples that require BSL-2 precautions. Because this in an upper-level course that requires prerequisite BSL-I-level lab activities, students were mostly familiar with BSL-I precautions. Nonetheless, during the first week, students received important safety training on proper BSL-I and BSL-2 procedures, including sterile techniques, pipetting, and plating of BSL-I Saccharomyces cerevisiae, and were required to demonstrate proficiency with these BSL-I procedures before performing BSL-2 procedures, including safe handling of potentially pathogenic unknown organisms (17). Students were required to wear personal protective equipment (gloves, lab coat, and eye protection) at all times and received instructions for how to minimize aerosolizing cultures (e.g., making sure lids are secured during vortexing and gently opening and handling culture tubes) and perform potential aerosol-generating procedures, such as pipetting and vortexing, in a biological safety cabinet. All bench surfaces and objects on the laboratory bench were disinfected after each class with $70 \%$ ethanol. The instructors were responsible for autoclaving all plates and contaminated materials after every class according to the minimal standards set by the American Society for Microbiology biosafety guidelines (17). All chemicals in this course are low-risk biohazardous agents except for methylene blue, hydrochloric acid, iodine, and iso-octane, which were discarded according to the institutional biohazard waste disposal guidelines.

All ingredients used for brewing were food grade, and brewing was conducted in a space safe for food handling. While many different types of wild yeast can be used for brewing, we were cautious to only use wild Saccharomyces cerevisiae. This activity and the associated research were submitted to the University of Arkansas Institutional Review Board (IRB) Committee (protocol number 1807/339/4) and were determined to be exempt. The course also included optional tours of a local craft brewery (Core Brewing in Springdale, AR) and a local homebrew store (Steve's Brew Shop in Fayetteville, AR) as well as an optional taste test of the final beers. We recognized that tasting of alcoholic beverages is a potentially sensitive subject, so we worked closely with the university administration to ensure that we complied with all university regulations and guidelines. We came up with the following guidelines for beer tasting: (i) tasting is entirely optional; any students who do not wish to participate do not have to, and the tasting will have no impact on student grades; (ii) only students 2 l years of age or older may participate in tasting; a valid photo ID with birth date will be required, and IDs will be checked by the trained staff at Core Brewing; (iii) tasting will only occur at Core Brewing; there will be no tasting of alcoholic beverages on campus; (iv) tasting will be through the sip and spit method only; there will be no drinking of the beer; (v) students must sign a waiver that includes the above information as well as a statement that they will act responsibly. 
TABLE I

Learning objectives and their corresponding methods of assessment

\begin{tabular}{l|l}
\hline \multicolumn{1}{l|}{ Learning objective } & Assessment \\
\hline I) Summarize and discuss primary research literature. & Homework, presentation, final paper, pre/postsurvey \\
\hline $\begin{array}{l}\text { 2) } \\
\text { Predict where wild yeast can be isolated based on the natural } \\
\text { ecology of yeast and explain how one can enrich for yeast from } \\
\text { environmental samples. }\end{array}$ & Homework, lab notebook, pre/postexam, pre/postsurvey \\
\hline 3) $\begin{array}{l}\text { Explain why and how internal transcribed space (ITS) sequencing is } \\
\text { used to determine fungal species and analyze ITS sequencing data to } \\
\text { assign the species of an unknown isolate. }\end{array}$ & Homework, lab notebook, pre/postexam, pre/postsurvey \\
\hline 4) $\begin{array}{l}\text { Describe the primary and secondary products of yeast fermentation } \\
\text { and how differences in fermentative metabolism across yeast strains } \\
\text { impact brewing. }\end{array}$ & Lab notebook, final paper, pre/postexam, pre/postsurvey \\
\hline 5) $\begin{array}{l}\text { Analyze yeast phenotypic data for traits relevant to brewing and then } \\
\text { use those data to predict brewing outcomes. }\end{array}$ & Lab notebook, final paper, pre/postexam, pre/postsurvey \\
\hline 6) $\begin{array}{l}\text { Explain the role of each ingredient and step in the brewing process } \\
\text { and scientifically design and implement a brewing protocol. }\end{array}$ & Lab notebook, final paper, pre/postexam, pre/postsurvey \\
\hline
\end{tabular}

DISCUSSION

\section{Field testing}

This class was developed and field tested through 2 years as an upper-level research-based undergraduate course at a 4-year public university, the University of Arkansas (23 students in 2017 and 24 students in 2018). Students worked independently for yeast isolation, in pairs for yeast sequencing and characterization, and in groups of four (two pairs) for brewing. Discussions within and between groups were encouraged. For yeast isolation, 37/47 students successfully isolated wild budding yeast. Based on ITS sequencing, 6/37 isolates were $S$. cerevisiae. Several other species were identified, including $S$. paradoxus, S. cariocanus, Pichia species (P. kudriavzevii, P. kluyveri, $P$. fermentans, and $P$. terricola), Meyerozyma caribbica, Lachancea fermentati, Wickerhamomyces anomalus, Kodamaea ohmeri, and Debaryomyces sp. Further characterization only proceeded with wild $S$. cerevisiae strains. While all-grain brewing may seem intimidating for novices, the "brew in a bag" method dramatically simplifies the process and works extremely well for the small volumes being brewed in the course. Neither the instructors nor most of the students had any experience brewing, but every group in both student cohorts was able to successfully brew beer.

In the second offering of the course, we changed the focus of the brewing module to be more "yeast centric." We did this by having pairs of lab partners (each working with either a commercial brewing strain or a wild S. cerevisiae strain) scientifically design a single brewing recipe to compete the yeast strains. While the first cohort of students still scientifically designed a brewing recipe, this change allowed each group of students to predict how the final beer would change depending on the properties of the yeast and then test these predictions in the final characterization of the beer.

Overall, student feedback on the course was highly positive. Anonymous online evaluations rated the course very highly on a I (very poor) through 5 (excellent) Likert-like scale, with a 2017 rating of $4.80 / 5$ (compared to a departmental mean of 3.96) and a 2018 rating of $4.79 / 5$ (compared to a departmental mean of 3.83). Student comments pointed to a particular appreciation of connecting molecular biology to real-world applications. The following are examples of student comments:

- I was able to learn about genetics through real-life situations and to apply what I learned in a way that made much more sense than my general genetics course ever did.

- This class is a great example of helping students to understand complex concepts by utilizing an interesting life application.

- Great reminder of some biology concepts that did not seem applicable to real life when taught in another course.

\section{Evidence of student learning}

Student learning was assessed using a variety of methods (Table I). Take-home problem sets or assignments (Appendix 5 in the supplemental material) were used to assess understanding of the assigned readings and certain learning objectives. For example, assignment number 2 focused on yeast isolation from the environment (learning objectives $\mathrm{I}$ and 2 , grade range $=50 \%$ to $98 \%$, mean $=85 \% ; n=24$ ), assignment number 3 focused on molecular characterization of yeast species (learning objectives I and 3, grade range $=67 \%$ to $100 \%$, 


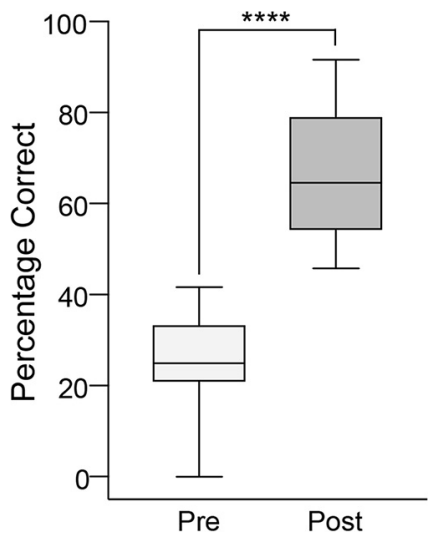

FIG 2. Pre- and postexams show significant gains in student learning. The box plot depicts the median and interquartile range, while the whiskers depict the range; ${ }^{* * *}, P=6 \times 10^{-14}$, two-tailed unpaired Mann-Whitney $U$ test.

mean of $89 \% ; n=24$ ), and assignment number 4 required students to write a brief literature review on the microbial ecology, physiology, and potential biotechnological applications for non-Saccharomyces cerevisiae yeast (learning objective I, grade range $=75 \%$ to $100 \%$, mean $=92 \% ; n=24$ ).

Lab notebook entries were used to assess students' abilities to understand the rationale for their experiments as well as their design, analyses, and interpretations. For notebook number I (yeast isolation, ITS PCR, and sequence analysis), the grade range was $73 \%$ to $100 \%$ with a mean of $91 \%(n=24)$. For notebook number 2 (yeast phenotypic characterization: fermentation rates, QPCR of genes responsible for ester and fusel alcohol production, and GCMS), the grade range was $62 \%$ to $100 \%$ with a mean of $89 \%$ $(n=24)$. For notebook number 3 (brewing recipe design and implementation plus characterization of final beers), the grade range was $80 \%$ to $100 \%$ with a mean of $93 \%(n=24)$.

Students were evaluated on their ability to present short (I0- to I5-min) mini-lectures on their choice of topics related to microbial fermentation (grade range of $84 \%$ to $100 \%$, mean $=89 \% ; n=24$ ). A final written report in the format of a primary research article was used to assess students' abilities to synthesize what they learned, especially in terms of understanding the entire brewing process and the scientific design of their brewing recipe (see rubric in Appendix 5). Grades on the final paper ranged from $50 \%$ to $98 \%$ (one assignment was incomplete), with a mean of $86 \%$. Overall, $22 / 24$ students received at least an acceptable passing grade $(70 \%)$ on the final paper, suggesting that they achieved learning objectives 4 and 5 .

We also measured changes in student learning with pre- and posttests, and we assessed changes in student perceptions of their skills and knowledge with pre- and postsurveys (see Appendix 5 for exam and survey questions). The average pretest score was $26 \%$ correct, which rose to $67 \%$ following participation in the course (Fig. $2 ; n=24$ in 2018). This was statistically significant $\left(P=6 \times 10^{-14}\right.$, twotailed unpaired Mann-Whitney $U$ test) and of large effect $($ Cliff's delta $=1)$. Additionally, students showed significant
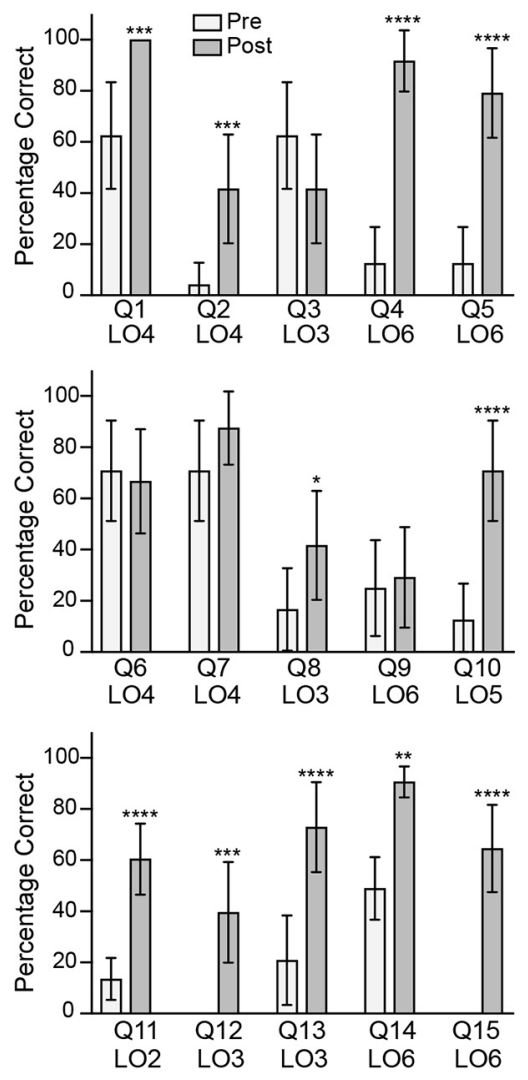

FIG 3. Individual item responses for pre- and postexam scores. Exam questions (QI to Q I5) can be found in Appendix 5 in the supplemental material. LO denotes the learning objectives; ${ }^{*}, P<$ 0.05 ; ${ }^{* *}, P<0.01$; ${ }^{* * *}, P<0.001$; ${ }^{* * * *}, P<0.000 I$, two-way ANOVA, Fisher's LSD test.

increases in learning for the majority of the questions (Fig. 3). We should note that formal assessment in the course did not include any exams, so these gains are more likely to reflect long-term understanding instead of shortterm memorization. Students also self-reported their perceptions of competency on pre- and postsurveys. Following the course, students showed significantly higher confidence in their abilities to isolate wild yeast from nature, use molecular biology and phylogenetics to identify yeast species, describe the major steps in brewing, and brew beer on their own (Fig. 4; $n=24$ in 2018). Coming into the class, students felt confident with reading scientific articles, although they may have still showed a small gain in confidence following the course $(P=0.08$, two-way analysis of variance [ANOVA], Fisher's least significant difference [LSD]).

\section{Possible modifications}

This course was designed and offered twice as an upperlevel course that met twice a week for one semester. There are several modifications that could be included for a shorter course. For example, the yeast isolation can be shortened by the instructor plating or streaking colonies from fermentation-positive cultures. Additionally, the brewing module can 


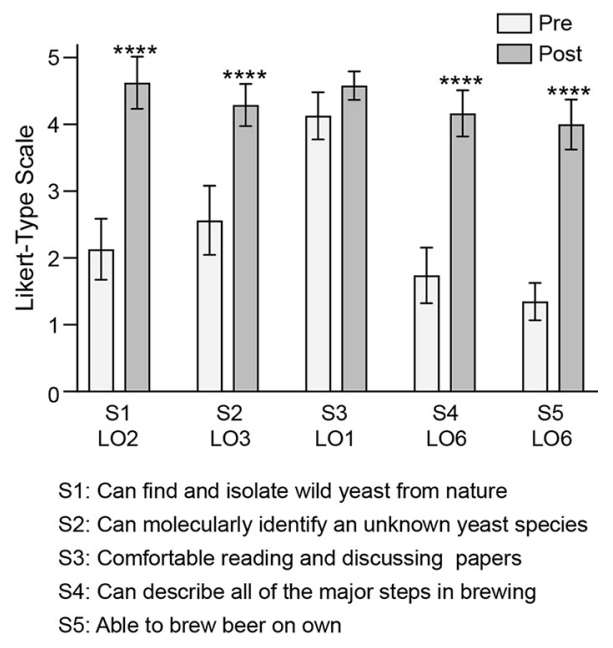

FIG 4. Pre- and postsurveys show increase in self-reported perceptions in student ability. Survey questions (SI to S5) can be found in Appendix 5. LO denotes the learning objectives; ${ }^{* * * *}$, $P<0.000$ l, two-way ANOVA, Fisher's LSD test.

be shortened by using commercial malt extracts instead of mashing whole grains. Optional activities that could be omitted include a guest lecture from a local craft brewer and tours of both a local craft brewery and homebrew store.

One of the optional modules we included was strain characterization of flavor compound formation (e.g., volatile esters and fusel alcohols), which we did both at the gene expression level via quantitative real-time PCR (qPCR) and directly via gas chromatography-mass spectrometry (GCMS). We understand that some instructors may not have access or funds to include these modules. One less expensive alternative to $\mathrm{QPCR}$ would be semiquantitative PCR (18). An alternative to GC-MS is sensory analysis, where students can be trained to identify esters and fusel alcohols by taste (individual flavor standards may also be purchased from FlavorActiV to facilitate compound identification). Future iterations of the course could include analysis of yeast growth under different conditions relevant to brewing (e.g., temperature and wort composition) or basic enzyme kinetics for the amylases during mashing.

\section{SUPPLEMENTAL MATERIAL}

Supplemental material is available online only.

SUPPLEMENTAL FILE I, DOCX file, 7.I MB.

\section{ACKNOWLEDGMENTS}

Funding for this project was supported, in part, by National Science Foundation grant number IOS-1656602. We are thankful to Ron Schmidt of Core Brewing and Distilling, LLC, for providing a guest lecture and a guided brewery tour.
We also thank Steve Wilkes for giving a guided tour of Steve's Brew Shop.

We declare no conflicts of interest.

\section{REFERENGES}

I. McGovern PE, Mirzoian A, Hall GR. 2009. Ancient Egyptian herbal wines. Proc Natl Acad Sci U S A 106:736I-7366. https://doi.org/10.1073/pnas.081 I578I06.

2. McGovern P, Jalabadze M, Batiuk S, Callahan MP, Smith KE, Hall GR, Kvavadze E, Maghradze D, Rusishvili N, Bouby L, Failla O, Cola G, Mariani L, Boaretto E, Bacilieri R, This P, Wales N, Lordkipanidze D. 2017. Early Neolithic wine of Georgia in the South Caucasus. Proc Natl Acad Sci U S A II4:EI0309EI03 I8. https://doi.org/10.1073/pnas. 17|4728II4.

3. McGovern PE, Zhang J, Tang J, Zhang Z, Hall GR, Moreau RA, Nuñez A, Butrym ED, Richards MP, Wang CS, Cheng G, Zhao Z, Wang C. 2004. Fermented beverages of pre- and proto-historic China. Proc Natl Acad Sci U S A 101:17593-17598. https://doi.org/10.1073/pnas.0407921102.

4. Hesseltine CW. 1979. Some important fermented foods of Mid-Asia, the Middle East, and Africa. J Am Oil Chem Soc 56:367-374. https://doi.org/10.1007/BF0267I50I.

5. Tamang JP, Watanabe K, Holzapfel WH. 2016. Review: diversity of microorganisms in global fermented foods and beverages. Front Microbiol 7:377. https://doi.org// 0.3389/fmicb.2016.00377.

6. Wang QM, Liu WQ, Liti G, Wang SA, Bai FY. 20I2. Surprisingly diverged populations of Saccharomyces cerevisiae in natural environments remote from human activity. Mol Ecol 21:5404-5417. https://doi.org/I 0.I I I I/j. I365-294X.20I2.05732.x.

7. Liu L, Wang J, Rosenberg D, Zhao H, Lengyel G, Nadel D. 2018. Fermented beverage and food storage in 13,000 y-old stone mortars at Raqefet Cave, Israel: investigating Natufian ritual feasting. J Archaeol Sci Rep 21:783-793. https://doi.org/ 10.1016/j.jasrep.2018.08.008.

8. Meussdoerffer FG. 2009. A comprehensive history of beer brewing, P I-42. In Eßlinger HM (ed), Handbook of brewing: processes, technology, markets. Wiley- $\mathrm{VCH}$, Weinheim, Germany.

9. Humia BV, Santos KS, Barbosa AM, Sawata M, Mendonça MDC, Padilha FF. 2019. Beer molecules and its sensory and biological properties: a review. Molecules 24:1568. https://doi .org/I0.3390/molecules2408I568.

10. Petruzzi L, Corbo MR, Sinigaglia M, Bevilacqua A. 2016. Brewer's yeast in controlled and uncontrolled fermentations, with a focus on novel, nonconventional, and superior strains. Food Rev Int 32:34I-363. https://doi.org/10.1080/ 87559I29.20I5.10752II.

II. Steensels J, Verstrepen KJ. 20I4. Taming wild yeast: potential of conventional and nonconventional yeasts in industrial fermentations. Annu Rev Microbiol 68:6I-80. https://doi.org/I0 .I |46/annurev-micro-09|2/3-I|3025.

12. Buck LB, Bretz SL, Towns MH. 2008. Characterizing the level of inquiry in the undergraduate laboratory. J Coll Sci Teach 38:52-58. 
13. Sampaio JP, Gonçalves P. 2017. Biogeography and ecology of the genus Saccharomyces, $\mathrm{P}|3|-153$. In Buzzini P, Lachance MA, Yurkov A (ed), Yeasts in natural ecosystems: ecology. Springer, Cham, Switzerland.

14. White TJ, Burns T, Lee S, Taylor J. 1990. Amplification and direct sequencing of fungal ribosomal RNA genes for phylogenetics, P 315-322. In Innis MA, Gelfand DH, Sninsky JJ, White TJ (ed), PCR protocols: a guide to methods and applications. Academic Press, New York, NY.

15. Bamforth CW, American Society of Brewing Chemists. 2006. Scientific principles of malting and brewing. American Society of Brewing Chemists, St. Paul, MN.
16. Haas GJ, Barsoumian R. 1994. Antimicrobial activity of hop resins. J Food Prot 57:59-6I. https://doi.org//0.43/5/0362028X-57.1.59.

17. Byrd JJ, Emmert E, Maxwell R, Townsend H, ASM Task Committee on the Revision of the 2012 Laboratory Biosafety Guidelines. 2019. Guidelines for biosafety in teaching laboratories version 2.0: a revised and updated manual for 2019. J Microbiol Biol Educ 20:20.3.57. https://doi.org//0.1 I28/jmbe .v20i3.1975.

18. Marone M, Mozzetti S, De Ritis D, Pierelli L, Scambia G. 200 I. Semiquantitative RT-PCR analysis to assess the expression levels of multiple transcripts from the same sample. Biol Proced Online 3:19-25. https://doi.org//0.1251/bpo20. 